\title{
POSITRON ANNIHILATION IN HELIUM
}

GPO PRICE

$\$$

CFSTI PRICE(S) \$

Hard copy (HC)

Microfiche (MF)

1.00

ff 653 July 65

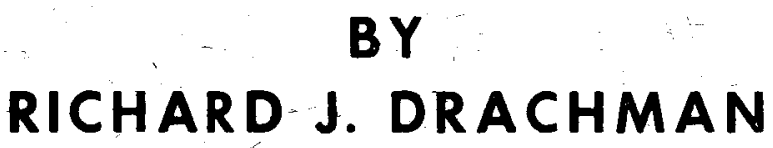

MAY 1966 


\title{
Positron Annihilation in Helium
}

\author{
Richard J. Drachman \\ National Aeronautics and Space Administration \\ Laboratory for Theoretical studies \\ Goddard Space Flight Center \\ Greenbelt, Maryland
}

The modified adiabatic scattering function previously used to compute phase shifts for positron-helium scattering below the positronium threshold is applied to the problem of annihilation in helium. A large enhancement factor relative to the Dirac rate is found, varying with energy from 2.30 to 3.16 . The probability of finding the spectator electron in states of the $\mathrm{He}^{+}$ion other than the ground state is computed and found to be small but probably observable. 
I. Positron Scattering Function

We have previously discussed the adiabatic approximation ${ }^{l}$ and applied it to the problem of low energy positron-helium scattering. ${ }^{2}$ Let us now consider its application to the computation of positron annihilation in helium.

The scattering wave function for a positron of momentum $\mathrm{k}$ has the form:

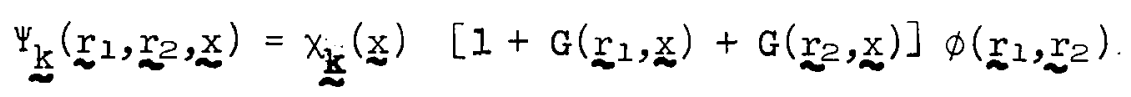

Here $\underset{x}{x}$ is the positron coordinate, and $\underline{\sim}_{1}, \underline{\sim}_{2}$ are the coordinates of the two electrons, all measured from the helium nucleus. The function $\phi$ describes the ground state of the helium atom. The asymptotic form of the function $\Psi$ will be correct if $x_{k} \rightarrow e^{i \frac{i k}{\alpha}} \cdot x$ since the correlation function $G$ vanishes for large values of $\mathrm{x}$, and the two electrons are assumed to be independently polarized. Taking the positron interaction as perturbation

$$
v=2 \sum_{i=1,2}\left[\frac{1}{x}-\frac{1}{\left|x-r_{i}\right|}\right] \text {, }
$$

one can evaluate $G(\underset{x}{x}, x)$ correct to first order in $V$ by the method of Dalgarno and Lewis $^{3}$. If we make the shielding approximation employed previously ${ }^{2}$

$$
\phi\left(\underline{\sim}_{1}, \underline{r}_{2}\right)=\pi^{-1} \beta^{3} \exp \left[-\beta\left(r_{1}+r_{2}\right)\right],
$$

the equation for $G(\underset{\sim}{\mathbf{a}}, \mathrm{x})$ becomes

$$
\nabla^{2} G-2 \beta \frac{d G}{d r}=2\left[\frac{1}{x}-\frac{1}{\mid x-\frac{r}{\alpha}}\right]-2 \beta e^{-2 \beta x}\left[1+\frac{1}{\beta x}\right] .
$$


This is similar to the equation solved by Dalgarno and $\mathrm{Lynn}^{4}$ for the case of hydrogen, and can be related to that result directly by making the change of variables $\underset{\sim}{y}=\beta r, z=\beta x$. Then one finds that $\beta G(y, z)$ satisfies the same equation as does the function derived by Dalgarno and $\mathrm{Iynn}^{4}$, and can be taken over from their work. They derived an expression, in elliptical coordinates, which contains implicitly all terms in the Legendre polynomial expansion

$$
G(y, z)=\sum_{\ell=0}^{\infty} g_{\ell}(y, z) P_{\ell}(\cos \alpha),[\cos \alpha=\hat{y} \cdot \hat{z}] .
$$

Our previous experience ${ }^{l}$ indicated that the monopole term in the expansion gives excessive short-range attraction (correlation), and in the following it will be completely suppressed. Then, in elliptical coordinates one has the modified correlation function for helium

$G(y, z)=\beta^{-1}\left[F(z, \lambda, \mu)-\frac{1}{2} \int_{-1}^{1} d(\cos \theta) F(z, \lambda, \mu)\right] \equiv \beta^{-1}\left[F-F_{O}\right]$.

Here, $\lambda=z^{-1}\left[y+\left(y^{2}+z^{2}-2 y z \cos \theta\right)^{1 / 2}\right], \quad 1 \leq \lambda \leq \infty$

$$
\begin{gathered}
\mu=z^{-1}\left[y-\left(y^{2}+z^{2}+2 y z \cos \theta\right)^{1 / 2}\right],-1 \leq \mu \leq 1, \\
\cos \theta=(1+\lambda \mu)(\lambda+\mu)^{-1}, \\
F(z, \lambda, \mu)=(\lambda+\mu) A+\left[1+2 A z^{-1}\right] \ln (1+\lambda)+D(z, \mu),
\end{gathered}
$$

where $D(z, \mu)=\left(1+z^{-1}\right)[E I(-z[1-\mu])-\ln (1-\mu)$

$$
\left.-e^{-2 z}(\operatorname{Ei}[z(I+\mu)]-\ln [I+\mu])\right],
$$




$$
E_{i}(-x)=-\int_{x}^{\infty} e^{-t} t^{-1} d t,
$$

and $\dot{A}=\frac{1}{z}\left[(1+z) e^{-2 z}-1\right]$.

\section{Annihilation Rate}

The rate of annihilation of positrons in helium is given by the expression ${ }^{5}$

$$
\lambda=\pi r_{O}^{2} c N z_{k}
$$

where $r_{0}$ is the classical electron radius $e^{2} / \mathrm{mc}^{2}, \mathbb{N}$ is the number density of atoms in the target gas, and $z_{k}$ is an effective electron number given by the expression

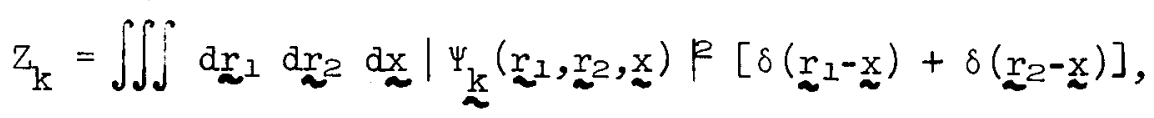

and provides a measure of the probability that the positron and an electron are at the same point in space. If one were to set $V=0$, thereby reducing the distortion of the atom as well as the positron scattering to zero, then

$$
\underset{\sim}{\Psi_{N}}=\phi e^{i \underline{i k} \cdot \mathrm{x}} .
$$

Thus the effective electron number $z_{k}=2$, and the annihilation rate goes over to the Dirac rate ${ }^{5}$ which is the quantum electrodynamic result for free particles. For our type of wave function,

$$
\frac{1}{2} z_{k}=\pi^{-1} \int d z e^{-2 z}\left|x_{k}(x)\right|^{2}\left[(1+G[z, z])^{2}+<G^{2}>\right],
$$

where

$$
\left\langle Q^{2}>\equiv \iint d r_{1} d r_{2} \phi Q \phi\right. \text {. }
$$


The energy independent parts of $\mathrm{z}_{\mathrm{k}}$ can be conveniently isolated by writing $x_{\underline{L}}(x)$ in a partial wave expansion

$$
X_{\underline{K}}(x)=\sum_{L=0}^{\infty} x^{-1} U_{L}(x) P_{L}(\cos \theta)(2 L+I) i^{L} e^{i \delta} L
$$

Using

$$
e^{i k \cdot x}=\sum_{L=0}^{\infty} j_{L}(k x) P_{L}(\cos \theta)(2 L+1) i^{L}
$$

one finds

$$
\begin{aligned}
& \int d \Omega\left|x_{\underline{k}}(x)\right|^{2}=\int d \Omega\left|e^{i \underline{k} \cdot x}+\left(x_{\underline{k}}(\underline{x})-e^{i \underline{k} \cdot \underline{x}}\right)\right|^{2} \\
& =4 \pi\left[I+\sum_{L=0}^{\infty}(2 I+1)\left(x^{-2} U_{L}^{2}-j_{L}^{2}\right)\right] \text {, }
\end{aligned}
$$

and

$$
\frac{1}{2} z_{K}=4 \int_{0}^{\infty} d z e^{-2 z} z^{2}\left[\left(I+G[z, z]+G^{2}\right\rangle\right]\left\{I+\sum_{L=0}^{\infty}(2 I+I)\left[\beta^{2} z^{-2} U_{L}^{2}\left(\frac{k z}{\beta}\right)-J_{L}^{2}(k z / \beta)\right]\right\}
$$

Thus, a rapidly converging series of energy-dependent corrections and a single energy independent term together yield the effective electron number. The functions $U_{\mathrm{L}}$ are obtained numerically from the solutions discussed previous $1 \mathrm{y}^{2}$, and have the asymptotic form

$$
\mathrm{U}_{\mathrm{L}}(\mathrm{x}) \rightarrow \mathrm{k}^{-1} \sin \left(\mathrm{kx}-\mathrm{In} / 2+\delta_{\mathrm{L}}\right)
$$


The problem thus requires the evaluation of $\left\langle G^{2}\right\rangle$ and $G(z, z)$ and the numerical integration of Eq. (14). In elliptical coordinates, $y=z$ is equivalent to $\lambda=\mu=1$, since $y=z$ and $\cos \theta=1$. For these values

$$
\begin{aligned}
F(z, 1,1) & =2 A+\left[1+2 z^{-1} A\right] \ln 2 \\
& +\left(1+z^{-1}\right)\left[\ln \gamma z-e^{-2 z}(\operatorname{Ei}[2 z]-\ln 2)\right],
\end{aligned}
$$

where $\ln \gamma=0.5771 \ldots$, Euler's constant.

The integral in Eq. (6) takes the form

$$
\begin{aligned}
& \frac{1}{2} \int_{-1}^{1} d(\cos \theta) F(z, \lambda, \mu) \lambda+\mu=2=A \int_{-1}^{1} d(\cos \theta) \\
& \quad+\left[1+2 z^{-1} A\right] \int_{1}^{3} d \lambda(\lambda-1) \ln (1+\lambda)+\int_{-1}^{1} d \mu(1-\mu) D(z, \mu) .
\end{aligned}
$$

Then, after performing the integrations, one obtains the result

$$
G(z, z)=\beta^{-1}\left\{\left[1+z^{-1}\right]\left[\ln 2 \gamma z-E i(-2 z)-1 / 2 z+e^{-2 z}(1+1 / 2 z)\right]-1\right\},
$$

which approaches zero as $\mathbf{z} \rightarrow 0$, confirming the removal of the short-range parts of the correlation. Some numerical values of $(I+G)^{2}$ are shown in Table I.

The remaining term required to evaluate the annihilation rate is $\left\langle G^{2}\right\rangle$ which has been evaluated numerically from the formula 


$$
\begin{gathered}
-7- \\
\left\langle G^{2}\right\rangle=\beta^{-2}\left[\left\langle F^{2}\right\rangle-\left\langle\mathrm{F}_{0}^{2}\right\rangle\right] .
\end{gathered}
$$

It also vanishes at $\mathrm{z}=0$ and decreases as $\mathrm{z}^{-4}$ for large $\mathrm{z}$. For $\beta=1.5992$ it is also exhibited in Table I. In Fig. $I$ and Table II the values of $\frac{1}{2} Z_{k}$ are shown over the energy range from zero to the positronium threshold. These have been computed numerically, using one set of solutions to the scattering $[\beta=1.5992, \alpha=0]$ discussed previously ${ }^{2}$, but including only the $I=0$ part of the sum over partial waves. We estimate that the neglect of $I>0$ contributes an error of less than $2 \%$ to the annihilation rate, and the error vanishes for $k \rightarrow 0$. The rates obtained are very high $^{6}$, and exhibit considerable variation with energy, which should soon be observable experimentally. ${ }^{7}$

\section{Excitation of the Residual Ion}

Suppose that an electron is annihilated when the positron is at position x. The annihilation takes place very rapidly compared with atomic times, and so the subsequent behavion of the residual $\mathrm{He}^{+}$ion can be treated by the sudden approximation ${ }^{8}$. The probability that the $\mathrm{He}^{+}$ion will be in the state whose wave function is $\phi_{n}$ is then given by

$$
P_{n}=\int d \underset{\sim}{d}\left|a_{n}(\underset{x}{x})\right|^{2} / \sum_{n} \int d x\left|a_{n}(x)\right|^{2}
$$

where $a_{n}(\underset{\sim}{x})=\int d \underset{\sim}{d r} \phi_{n}^{*}(\underset{\sim}{r}) \Psi_{\underset{\sim}{k}}(\underset{\sim}{x}, \underset{\sim}{x}, \underset{\sim}{r})$.

The denominator in Eq. (19) is 


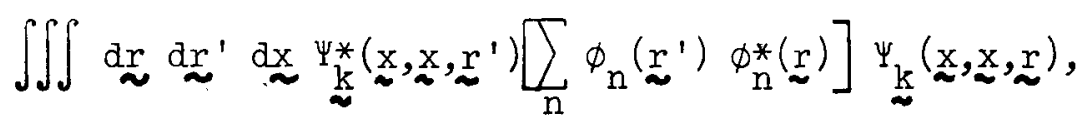

and since $\left\{\phi_{n}\right\}$ is a complete set of one-electron functions, closure can be applied to the sum in Eq. (20). Thus

and

$$
\begin{aligned}
P_{n} & =\int d x\left|a_{n}(x)\right|^{2} / \iint d \underset{\sim}{d r}\left|\Psi_{x}(x, x, r)\right|^{2} \\
& =\int d x\left|a_{n}(x)\right|^{2} / \frac{1}{2} z_{k}, \\
& \sum_{n} P_{n}=1 .
\end{aligned}
$$

If the probability for exciting states other than the ground state is not too small, there may be a possibility of observing the radiative deexcitation of the residual $\mathrm{He}^{+}$ion, following annihilation. A favorable transition might be the $2 \mathrm{p} \rightarrow 1 \mathrm{~s}$ ultraviolet line at $304 \stackrel{\circ}{\mathrm{A}}$, and we now proceed to compute its intensity, using the modified adiabatic wave function.

From Eqs. (1) and (19) one obtains

$$
\begin{aligned}
& P_{n}=2\left(\pi Z_{K_{k}}\right)^{-1} \int d z_{\sim} e^{-2 z}\left|x_{\underline{\alpha}}\right|^{2}\left[(1+G[z, z]) Q_{n}+W_{n}(z)\right]^{2} \\
& \text { where } \quad Q_{n}=\left(\beta^{3} / \pi\right)^{1 / 2} \int d \underset{\sim}{r} e^{-\beta r} \phi_{n}(\underline{r}) \\
& W_{n}(z)=\left(\beta^{3} / \pi\right)^{1 / 2} \int \underset{\sim}{d} G(\underset{\sim}{2}, z) \phi_{n}(\underset{\sim}{r}) .
\end{aligned}
$$

For excitation to the $2 p$ state of the ion $Q_{n}=0$ and only the dipole part of $G$ contributes to $W_{n}(z)$. This is obtained by inserting Eq. (5) into Eq. (4) and yields 


$$
\begin{aligned}
g_{1}(y, z) & =\beta^{-1}\left\{z^{-2}\left[y+y^{2} / 2\right]+C_{1}\left[e^{-2 y} / 2 y^{2}+\left(1+y^{-1}+\frac{1}{z} y^{-2}\right)\right]\right\} & \text { for } y<z \\
& =\beta^{-1}\left\{z+C_{2}\left[1+y^{-1}+\frac{1}{z y^{-2}}\right]\right\} &
\end{aligned}
$$

for $y>z$

where

$$
\begin{aligned}
& C_{1}=\frac{3}{2} e^{-2 z}\left(1+z^{-1}\right)^{2}, \\
& C_{2}=C_{1}-z+\frac{3}{2}\left(1-z^{-2}\right),
\end{aligned}
$$

and then

$$
W_{2 p}(z)=\frac{4}{3} \beta^{-5 / 2} \int_{0}^{\infty} d y y^{3} e^{-\left(I+\beta^{-1}\right) y} g_{1}(y, z) .
$$

The probability $\mathrm{P}_{2 p}$ has been computed for the same parameters as before and is found to be essentially independent of positron energy, (for $\mathrm{L}=0$ scattering) and equal to $0.82 \%$. Thus at least $0.82 \%$ of all annihilations should be followed by the $2 p \rightarrow$ ls transition. In addition, however, the experimentally observable radiation should include the effect of cascades from other states which pass through the $2 p$ state. S-states should dominate the cascade process. For S-states, $\mathrm{W}_{\mathrm{n}}=0$ since no monopole term is contained in $\mathrm{G}$. The Sstate probabilities are

$$
P_{n s}=[1+R]^{-1} Q_{n s}^{2}
$$

where $\quad R=\int d \underset{\sim}{d z} e^{-2 z}\left|x_{k}\right|^{2}<G^{2}>/ \int d z \underset{\sim}{ } e^{-2 z}\left|x_{k}\right|^{2}[1+G]^{2}$. 
Invoking closure over S-states, one finds that $\mathrm{P}($ all $\mathrm{S})=[\mathrm{I}+\mathrm{R}]^{-1}=99 \%$ where $R$ has been numerically evaluated, and proves to be essentially energy-independent. Thus $99 \%$ of all annihilations give s-states of the ion, and $0.82 \%$ go to the $2 \mathrm{p}$ state, leaving a negligible fraction in the higher $\mathrm{p}$ or $\mathrm{I}>1$ states. The partition among the various discrete $\mathrm{S}$-states is then given by $Q_{n}{ }^{2}$. $A$ general formula due to Mittleman ${ }^{9}$ is

$$
Q_{n}^{2}=(8 \beta / n)^{3}(2-\beta)^{2}(\beta-2 / n)^{2 n-4}(\beta+2 / n)^{-(2 n+4)},
$$

which was used to prepare Table III, which also shows the branching ratios 10 to the $2 p$ state. One finally finds that $1.2 \%$ of all annihilations should be followed by the $2 p \rightarrow$ Is transition. In addition, if a sufficiently strong electric field is applied to the target gas, the metastable 2 s level will mix with the $2 p$ level $^{11}$, and an increase in the $2 p \rightarrow$ ls transition rate to $3.2 \%$ of the annihilation rate will occur, and should be observable.

\section{Discussion}

We have presented above a calculation of the energy-dependent annihilation rate of positrons incident on a helium target, as well as a prediction of the intensity of the $\mathrm{He}^{+}$ultraviolet radiation which is expected to follow the annihilation. In this work a simple model wave function describes both processes, as well as the elastic scattering. The predictions are thus not expected to be exact, but previous experience has given some cause for optimism.

Nevertheless the results derived here must be used cautiously, as a guide only, until such time as more accurate solutions of the positron-helium scattering problem become available. As an example, we may note that the value of 
the parameter $\beta=1.5992$ used throughout has been chosen to produce agreement with the long-range $\mathrm{x}^{-4}$ potential 12 , and thus does not necessarily represent well some of the other properties of the system. In a somewhat different context, Mittleman ${ }^{9}$ has used a five-term exponential approximation to describe the helium atom wave function, and finds that a more accurate value of the $2 \mathrm{~s}$ excitation probability is $2.23 \%$, rather than our $2.00 \%$. This agreement indicates that our qualitative results should be used as preliminary estimates in designing experiments, which may eventually test the future detailed theories. 


\section{$\underline{\text { References and Footnotes }}$}

1) R. J. Drachman, Phys. Rev. 138, Al582 (1965).

2) R. J. Drachman, Phys. Rev. 144, 25 (1966).

3) A. Dalgarno and J. T. Iewis, Proc. Roy. Soc. (London) A233, 70 (1955).

4) A. Dalgarno and N. Lynn, Proc. Phys. Soc. (London) A70, 223 (1957).

5) R. A. Ferrell, Revs. Mod. Phys. 28, 308 (1956).

6) The rates are about twice as large as those obtained by H.S.W. Massey, J. Lawson and D. G. Thompson [preprint, 1966] who have not attempted to include the correlations at all, and who have used an adiabatic potential with dipole parts only in their scattering equation. Their results should seriously underestimate the correct annihilation rates.

7) W. R. Falk, P. H. R. Orth and G. Jones, Phys. Rev. Letters 14, 447 (1965). 8) I. I. Schiff in Quantum Mechanics, (McGraw-Hill Book Co., Inc., New York, 1955) 2nd ed., pp. 213-220.

9) M. H. Mittleman, Phys. Rev. Letters 16, 498 (1966). See also Ref. 11, which includes a one-term calculation like ours, but with $\beta=27 / 16$.

10) H. A. Bethe and E. E. Salpeter in Quantum Mechanics of One-and Two Electron Atoms, (Academic Press, Inc., New York, 1957), p. 266.

11) W. E. Lamb and M. Skinner, Phys. Rev. 78, 539 (1950). This paper contains a derivation of the dipole radiative lifetime of the metastable 2 s state of helium in the presence of an electric field, $T=1.6 \times 10^{-2} \mathrm{E}^{-2}$, with $\mathrm{E}$ in volts $/ \mathrm{cm}$. If this lifetime is shorter than that for collisional deexcitation, enhancement of the $304 \stackrel{\circ}{A}$ line occurs. A measurement of the latter value could in principle be made this way.

12) See Ref. 2, where another choice of $\beta$ is made. As Iong as full monopole suppression is maintained the results should be similar. The inclusion of shortrange monopole parts in $G$ will increase the effective electron number, and will reduce the relative excitation of the $2 p$ state of the ion. 
Table I. Numerical values of correlation functions required for the calculation of the positron-helium annihilation rate, for full monopole suppression and $\beta=1.5992$.

\begin{tabular}{c|c|c}
$\mathrm{z}$ & {$[\mathrm{I}+\mathrm{G}(\mathrm{z}, \mathrm{z})]^{2}$} & $\left\langle\mathrm{G}^{2}\right\rangle$ \\
\hline 0 & 1 & 0 \\
1.0 & 1.886 & .0230 \\
1.5 & 2.733 & .0473 \\
2.0 & 3.447 & .0495 \\
2.5 & 4.028 & .0397 \\
3.0 & 4.503 & .0282 \\
3.5 & 4.900 & .0188 \\
4.0 & 5.241 & .0123 \\
4.5 & 5.541 & .0080 \\
5.0 & 5.810 & .0053 \\
& 6.055 & .0036
\end{tabular}


Table II. Effective electron number for annihilation at selected values of incident positron energy. The Dirac rate corresponds to $\frac{1}{2} Z_{k}=1$.

\begin{tabular}{c|c} 
Energy $(\mathrm{eV})$ & $\frac{1}{2} \mathrm{z}_{\mathrm{k}}$ \\
\hline 0 & 3.16 \\
0.54 & 2.79 \\
1.22 & 2.58 \\
2.17 & 2.43 \\
3.40 & 2.35 \\
4.90 & 2.31 \\
6.66 & 2.30 \\
8.70 & 2.33 \\
11.0 & 2.38 \\
13.6 & 2.44 \\
17.8 & 2.54
\end{tabular}


Table III. Population and branching ratios following annihilation. The probability for exciting the various $\mathrm{S}-$ states of $\mathrm{He}^{+}$listed in column 1 are shown in column 2, and the fraction of each which eventually reaches the $2 p$ state is shown in column 3. [Cascades like $5 s \rightarrow 4 p \rightarrow$ $3 s \rightarrow 2 p$ are included, but are very small]. Column 4 gives the observable population of the $2 p$ state due to each S-state.

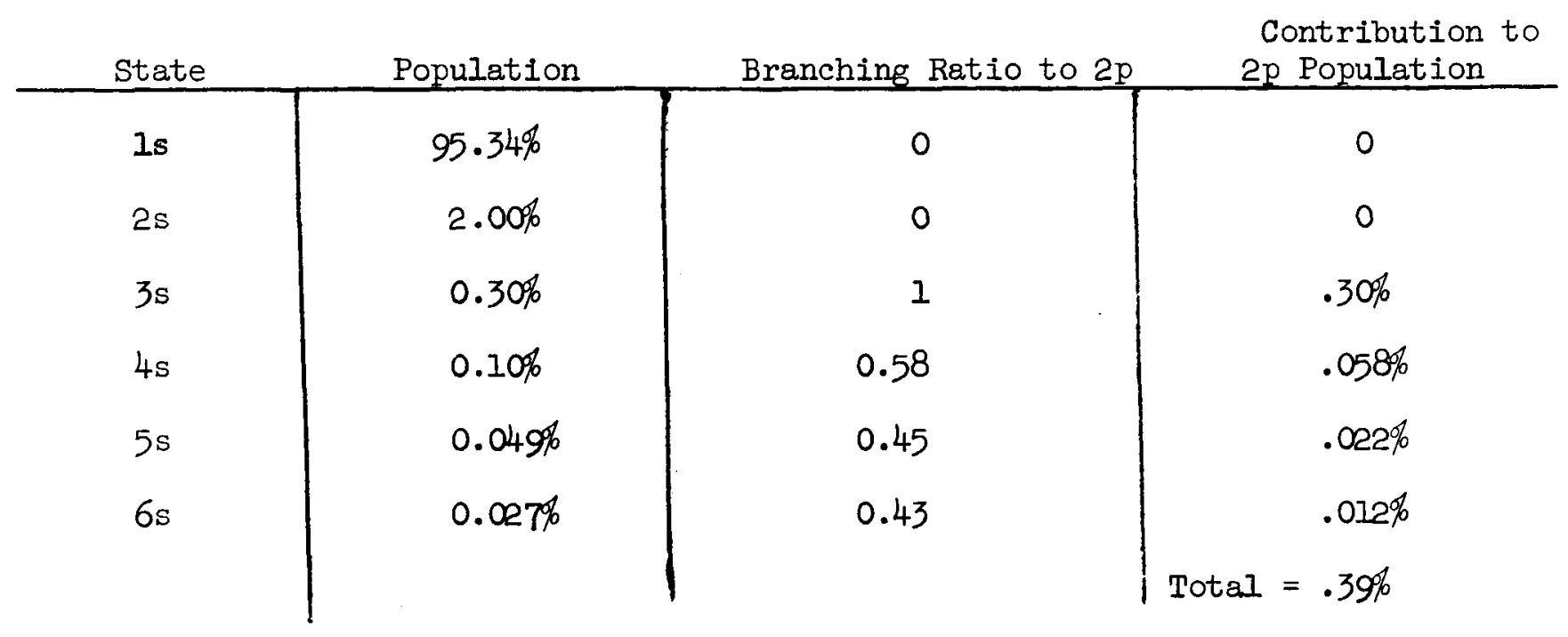




\section{Figure Captions}

Fig. 1. Effective electron number for annihilation versus incident positron energy in $\mathrm{eV}$. The Dirac rate corresponds to $1 / 2 \quad z_{k}=1$. 


$$
D
$$

